\title{
Intégration des TICE dans l'enseignement des Sciences de l'Ingénieur dans la filière Sciences Technologies Mécaniques aux lycées du Maroc : Réalité et Obstacles
}

\author{
Abderrahim Abouzaid, PhD \\ Mohamed. Taoufik, PhD \\ Ahmed. Moufti, PhD \\ Abdelhak. Lamsalmi, PhD
}

Centre Régional des Métiers de l'Education et de la Formation

Casablanca- Settat- Centre provincial de Settat, Maroc

doi: 10.19044/esj.2017.v13n13p476 URL:http://dx.doi.org/10.19044/esj.2017.v13n13p476

\begin{abstract}
Information and communication technology (ICT) in education has currently become an unavoidable pedagogical tool in any educational system of high standards. Its integration signifies a change in the learning processes and knowledge transmission and acquisition. In this article, we present the results of an exploratory research that we led with engineering science teachers of the Mechanical Science and Technology stream in high schools belonging to two regional Moroccan academies (Grand Casablanca, Settat and the Oriental). Our study focuses on the identification of the unknown current state of affairs and related to the integration of ICT in this subject of secondary education of the Mechanical Science and Technology stream in Moroccan high schools.

The results have shown that $95 \%$ of the interrogated teachers displayed a big interest towards the usage of ICT in their teaching practices whilst only $22.5 \%$ of them already integrate it regularly in the classroom. This paradox is chiefly due to a shortage of equipment within the institution, the lack of software and applications suitable for E.S as well as teachers' qualification and training. We also estimate that this research's results could prove useful to other streams while the integration of ICT in the training of future teachers and in the practices of the classroom still remains problematic.
\end{abstract}

Keywords: Education, Engineering science (E.S), Mechanical Science and Technology(MST), Training, Integration, ICT. 


\section{Résumé}

Les Technologies d'Information et de Communication en Education (TICE) sont devenues actuellement des outils pédagogiques incontournables dans tout système éducatif de qualité. Leur intégration signifie un changement de taille dans nos rapports au savoir et aux modes de sa transmission et de son acquisition. Dans cet article, nous présentons les résultats d'une recherche exploratoire que nous avons menée auprès des enseignants des sciences de l'ingénieur S.I de la filière Sciences Technologies Mécaniques (STM), dans deux académies régionales marocaines (Grande Casablanca-Settat et l'Orientale). Notre étude s'intéresse à l'identification de l'état du lieu méconnu de nos jours et relatif à l'intégration des TICE dans cette discipline de l'enseignement secondaire de la filière STM aux lycées du Maroc.

Les résultats ont montré que $95 \%$ des enseignants interrogés manifestent un grand intérêt quant à l'utilisation des TICE dans leurs pratiques pédagogiques alors que seulement $22.5 \%$ d'entre eux les intègrent de façon régulière en classe. Ce paradoxe est dû principalement à l'insuffisance des équipements matériels au sein des établissements, le manque de logiciels et d'applications appropriés aux programmes des S.I, et enfin la qualification et la formation des enseignants. Nous estimons aussi que ses résultats pourraient s'avérer utiles aux autres filières dès lors que l'intégration des TICE dans la formation des futurs enseignants et dans les pratiques de la classe demeure encore problématique.

Mots clés : Enseignement, Sciences de l'Ingénieur(SI), Sciences Technologies Mécaniques(STM), Formation, Intégration, TICE.

\section{Introduction}

$\mathrm{Au}$ cours des dernières années, l'usage des technologies de l'information et de la communication dans l'enseignement TICE au Maroc s'est accru d'une manière notoire, notamment dans le domaine de l'enseignement-apprentissage des sciences [1].

Cette évolution est en partie due aux discours prometteurs des spécialistes en informatique, des institutions gouvernementales, des économistes et des pédagogues [2]. Il s'avère donc impératif pour les enseignants d'acquérir les compétences nécessaires à un usage efficace des TICE dans leur pratiques enseignantes. Il en est de même pour les différentes institutions chargées de la formation des enseignants de s'assurer que ces derniers soient compétents dans ce domaine.

Les arguments de cette décision sont tels que les TICE permettent à l'enseignant d'atteindre ses objectifs pédagogiques en exploitant une plus grande variété d'approches. Les plus fervents de ses défenseurs sont 
convaincus que cela contribue à un changement positif du comportement et de la manière dans la pratique pédagogique des enseignants. Biaz et al. [3] ont étudié l'intégration des TICE dans le travail des enseignants et ont souligné l'importance de la formation pédagogique des enseignants à l'utilisation optimale des TICE pour l'amélioration de la qualité de l'enseignement et l'aisance d'apprentissage des élèves.

De nombreuses recherches ont étudié l'intégration des TICE dans l'enseignement de façon générale. Cleary et al. [4] ont analysé les facteurs déterminants de l'intégration des TICE en classe. Ils ont distingué cinq facteurs qui favorisent cette intégration et qui ont un impact positif sur les résultats scolaires des élèves :

- La formation ;

- $\quad$ Le contexte environnemental ;

- $\quad$ Les variables individuelles ;

- L L'importance d'une communauté et d'un réseau humain de soutien ;

- $\quad$ Le temps que l'on est disposé à y consacrer.

Bibeau en 2007[5] a étudié de son côté les conditions qui peuvent agir sur la réussite de l'intégration des TICE dans l'éducation de façon générale et conclu que les TICE améliorent la motivation des élèves et permettent le développement des opérations cognitives d’ordre supérieur. Cela a été confirmé par Labrique et ses co-auteur [6] que ces outils numériques peuvent contribuer à une formation moderne et efficiente des sciences de l'ingénieur S.I. Ils permettent aussi de faciliter l'acquisition rapide de compétences intermédiaires allant de la simple connaissance de la matière aux compétences de haut niveau.

Quant aux travaux de Karsenti et Gauthier [7] ont mis l'accent sur les principaux obstacles qui entravent l'intégration des TICE dans l'enseignement. Ces obstacles sont regroupés en deux grandes catégories :

- Les facteurs externes (liés à l’école, à la société, etc.).

- $\quad$ Les facteurs internes (liés à l'enseignant ou à l'enseignement).

En 2011, El Ouidadi et al. [8] ont conclu que la carence en formation (initiale ou/et continue) des enseignants pour ce qui est de l'usage des outils numériques, la déficience de l'exploitation pédagogique des TICE et les facteurs individuels sont les principaux obstacles liés à l’intégration des TICE en classe au Maroc.

Dans le contexte Marocain, l'émergence des nouvelles technologies et la manifestation accrue du rôle qu'elles semblent pouvoir jouer dans l'enseignement des Sciences de l'Ingénieur SI de la filière Sciences Technologies Mécaniques STM, un nouvel environnement technopédagogique est en train de s'implanter dans le champ éducatif afin de s’intégrer harmonieusement dans la société de l’information et du savoir. 
Les TICE permettent aussi de s'ouvrir sur un nouveau monde d'informations et de connaissances. Elles offrent ainsi aux utilisateurs l’opportunité d'aller chercher facilement les savoirs programmés plutôt que de les attendre de l'enseignant, surtout quand il s'agit d'une filière technologique telle que la STM dont les élèves ont besoin d’accéder aux informations les plus actuelles et aux données technologiques et scientifiques mises à jour afin de mieux répondre aux exigences de leurs apprentissages.

Depuis 1999, le Maroc a entamé ce processus d’intégration des TICE dans son système éducatif par l’opérationnalisation de la Charte nationale et de la formation. Cette charte a été consacrée à l'utilisation des Nouvelles Technologies de l'Information et de la Communication (NTIC) [9]. En 2005, le Ministère de l'éducation nationale a lancé le programme GENIE qui vise la généralisation des TICE. En 2008, des études ont montré que la sensibilisation, la communication, la responsabilisation, la révision et l'actualisation de la formation en termes de logistique, d'encadrement, d'accompagnement et de contenus sont nécessaires et indispensables pour l'avancement du programme GENIE [10]. Ce constat a incité le Ministère de l'éducation nationale et de la formation professionnelle au Maroc à le relancer dans son Plan d'urgence (2009-2012), qui intègre une stratégie visant à promouvoir, faciliter, et mettre en valeur une culture pédagogique favorisant l'utilisation des TICE dans l'enseignement - apprentissage. Il est à noter que jusqu’à nos jours le sujet est méconnu sur le plan de la filière STM.

C'est dans cette optique que le présent travail est réalisé afin d'identifier et d'analyser la situation actuelle des TICE dans l'enseignement de la discipline SI de la filière STM, de mettre en lumière ses réalités et ses obstacles, d'identifier les attentes relatives au sujet et enfin d'amorcer au moins la recherche, négligée jusqu’à maintenant, sur l'intégration des TICE dans l'enseignement technique de l'éducation nationale en général et dans la filière STM en particulier.

\section{Cadre théorique et Problématique}

L’enseignement des sciences de l’ingénieur aux lycées marocains, selon les directives pédagogiques [11] du Ministère de l'Education Nationale Marocain a pour objectifs de :

- $\quad$ Permettre à l'élève d'avoir l'aptitude à se remettre perpétuellement en question et à accepter les changements ;

- $\quad$ Appréhender les changements et les bouleversements technologiques qui s’opèrent dans le monde de l'industrie ;

- $\quad$ Trouver pour un problème donné des solutions technologiques compétitives selon les critères de qualité ; 
- $\quad$ Maitriser les outils méthodologiques de conception, de réalisation et de qualification d'un produit industriel.

La mise en œuvre du programme des sciences de l'ingénieur de la filière STM au lycée, s'effectue en 4 semestres à raison de deux semestres par niveau ( $1^{\text {ere }}$ année, $2^{\text {ème }}$ année). L’horaire des SI étant de 9 heures par semaine par année, répartie pour chaque niveau entre l'unité de conception (4heures), l'unité de production (4heures) et l'unité de CFAO (1heure). L'apprentissage est principalement conduit en s'appuyant sur les activités pratiques(AP) sous forme de travaux pratiques expérimentaux (TP-Ex), basés sur l’observation, la réflexion et la mise en évidence des lois scientifiques et techniques.

L’AP se déroule dans des laboratoires ayant des équipements adaptés aux unités enseignées :

- $\quad$ Unité de conception regroupe deux laboratoires :

- Le laboratoire des études des systèmes,

- Le laboratoire de CAO (logiciels de conception et modeleur 3D).

- $\quad$ Unité de réalisation concerne trois laboratoires :

- Le laboratoire d'expérimentation ;

- Le laboratoire de production ;

- Le laboratoire de CFAO (utilise des logiciels de simulations et de pilotage automatique des machines outils comme par exemple le « featureCAM ».

L’approche systémique adoptée par le programme de SI de la filière STM doit permettre de faire développer progressivement chez l'élève trois compétences :

- $\quad$ Compétence d’ordre méthodologique ;

- $\quad$ Compétence d'ordre intellectuel ;

- $\quad$ Compétence d’ordre communicationnel.

Le baccalauréat STM est un diplôme de formation académique débouchant essentiellement sur des filières d’ingénieries dans des écoles, facultés et instituts préparant des techniciens supérieurs, des licences, des masters et des diplômes d’ingénieurs destinés aux milieux industriels. Il permet aussi au candidat de s’intégrer directement à la vie professionnelle.

\section{Les TICE du point de vu institutionnel «La charte Nationale d'Education et de Formation/Programme GENIE/Plan d'urgence »}

Le Maroc a engagé une reforme de son système éducatif par l'opérationnalisation de la Charte nationale d'éducation et de formation. Dans cette charte, le levier 10 dans l'espace III est consacré à l'utilisation des nouvelles technologies de l'information et de la communication [9]. Dans cette optique, le Ministère de l'éducation nationale a lancé en 2005 le 
programme GENIE qui vise la généralisation des TICE [12]. Après la première phase, et pour remédier aux dysfonctionnements et déperditions constatés lors du déploiement de cette stratégie initiale, la direction du programme GENIE a lancé, dans le cadre du plan d'urgence (2009-2012), une nouvelle stratégie visant à promouvoir, faciliter et mettre en valeur une culture pédagogique qui favorise l'intégration des TICE dans l'enseignement et l'apprentissage. Ce nouveau souffle donné à la stratégie initiale réaffirme la volonté du ministère de créer un climat propice à l'utilisation des TICE dans les pratiques pédagogiques en classe. La feuille de route actuelle de ce programme prévoit, en plus des axes dédiés à l'infrastructure et à la formation, l'acquisition de ressources numériques éducatives de qualité adaptées au contexte culturel et scolaire marocain et le développement des usages des TICE [12].

La Charte Nationale d’Education et de Formation de son côté a fixé comme objectif la mise en place d'un centre informatique et d'une bibliothèque multimédia dans chaque établissement au terme de la décennie de l'éducation. En 2005, le programme GENIE a été mis en place en vue de répondre à ces préconisations. Les objectifs ont été assignés dans ce programme, visant la généralisation des technologies de l'information et de la communication pour leur intégration dans le Système d'Éducation et de Formation sont :

- L'infrastructure ;

- La formation des enseignants ;

- Le développement des contenus et l'adaptation des curricula à l'introduction des TICE.

Dans ce sens, le programme a envisagé diverses situations pour l'intégration des TICE dans la filière STM par le biais des recommandations suivantes :

o L'emploi de l'outil informatique comme moyen de situation et d'apprentissage (CAO, FAO, CFAO, GPAO, etc.).

o L'utilisation de l'Internet pour accéder à des données actualisées nécessaires à la formation ;

o L'utilisation de l'Internet comme moyen de recherche d'informations utiles pour le projet personnel de l'élève ;

o L'échange d'information et d'expérience avec d'autres personnes partageant les mêmes centres d'intérêt,

o La collaboration avec les entreprises et les divers organismes s'intéressant au domaine de la technologie [11]. 
Peu de recherches ont traité des pratiques et des obstacles liés à l'intégration des TICE dans l'enseignement des SI de la filière STM au Maroc, constat qui nous a interpelés pour poser la problématique de recherche tournant autour des axes suivants :

- $\quad$ La place des NTIC dans l’opération enseignement-apprentissage de la discipline SI de la filière STM aux lycées du Maroc (état des lieux).

- Les contraintes et les obstacles qui entravent l'intégration des TICE dans l’acte d'enseignement-apprentissage de la discipline SI.

\section{Méthodologie}

Cette étude est réalisée dans les académies régionales de l'éducation et de la formation (AREF), en l'occurrence, l'AREF Casablanca-Settat et l'Orientale durant l’année scolaire 2014/2015.

La population ciblée par notre étude est constituée de 60 enseignants du secondaire qualifiant de la filière STM, ce qui représente $27 \%$ de la population totale au niveau national [13]., répartis selon le tableau 1 :

\begin{tabular}{|c|c|c|c|}
\hline \multirow{2}{*}{ AREF } & \multicolumn{2}{|c|}{ Casablanca-settat } & \multirow{2}{*}{ Oriental } \\
\cline { 2 - 3 } & Grande Casa & Settat & 15 \\
\hline $\begin{array}{c}\text { Nombre } \\
\text { d'enseignants }\end{array}$ & 30 & 15 & 15 \\
\hline
\end{tabular}

Tableau 1 : Répartition de l'échantillonnage par AREF

Il est à noter que les enseignants impliqués dans cette étude possèdent 10 à 20 ans d'expérience, ayant une tranche d'âge entre 35 et 52 ans. La collecte des donnés a été effectuée au moyen d’un questionnaire anonyme, incluant des questions à réponses fermées et à choix multiples se rapportant à chacune des parties de la problématique de l'étude, elles sont conçues autour de quatre points suivants :

$>\quad$ Usages personnels des ressources numériques ;

$>\quad$ Usages professionnels des ressources numériques ;

$>\quad$ Représentation des enseignants de la discipline SI de la filière

STM sur l'utilité des TICE ;

Obstacles aux usages des TICE.

En plus des QCM de l'enquête, un certain nombre d'entretiens ont été effectués avec des enseignants de la discipline SI et des directeurs d'établissements objets de notre enquête. Le traitement du résultat est effectué par Excel.

\section{Résultats et Discussion}

Nous avons accordé un soin particulier à la détermination de l'échantillon. En effet, nous avons travaillé au plan d’échantillonnage probabiliste et, plus précisément, nous avons utilisé l'échantillonnage à plusieurs degrés. Nous avons distribué ce questionnaire de façon équitable 
dans les établissements scolaires appartenant aux académies en question, compte tenu de leurs emplacements géographiques.

\section{Usages personnels des ressources numériques :}

Les enseignants interrogés ont exprimé un grand intérêt pour l'environnement numérique; 5 \% seulement d'entre eux ne sont nullement intéressés par ces outils. Ces résultats montrent que les enseignants de la discipline SI de la filière STM présentent un grand intérêt pour l'usage des TICE (figure1). Cela a été constaté à travers les réponses se rapportant à l'usage personnel des TICE, 90\% des enseignants maitrisent les logiciels de bureautique (Word, Excel, PowerPoint...), d'autre part 47,5\% utilisent les moyens de communication (Internet, Email, Forum...), mais les enseignants qui exploitent les logiciels d'édition des pages Web (Dreamweaver, FrontPage...) et d'images (Photoshop, Premier...) ne représentent respectivement que $6 \%$ et $4 \%$.

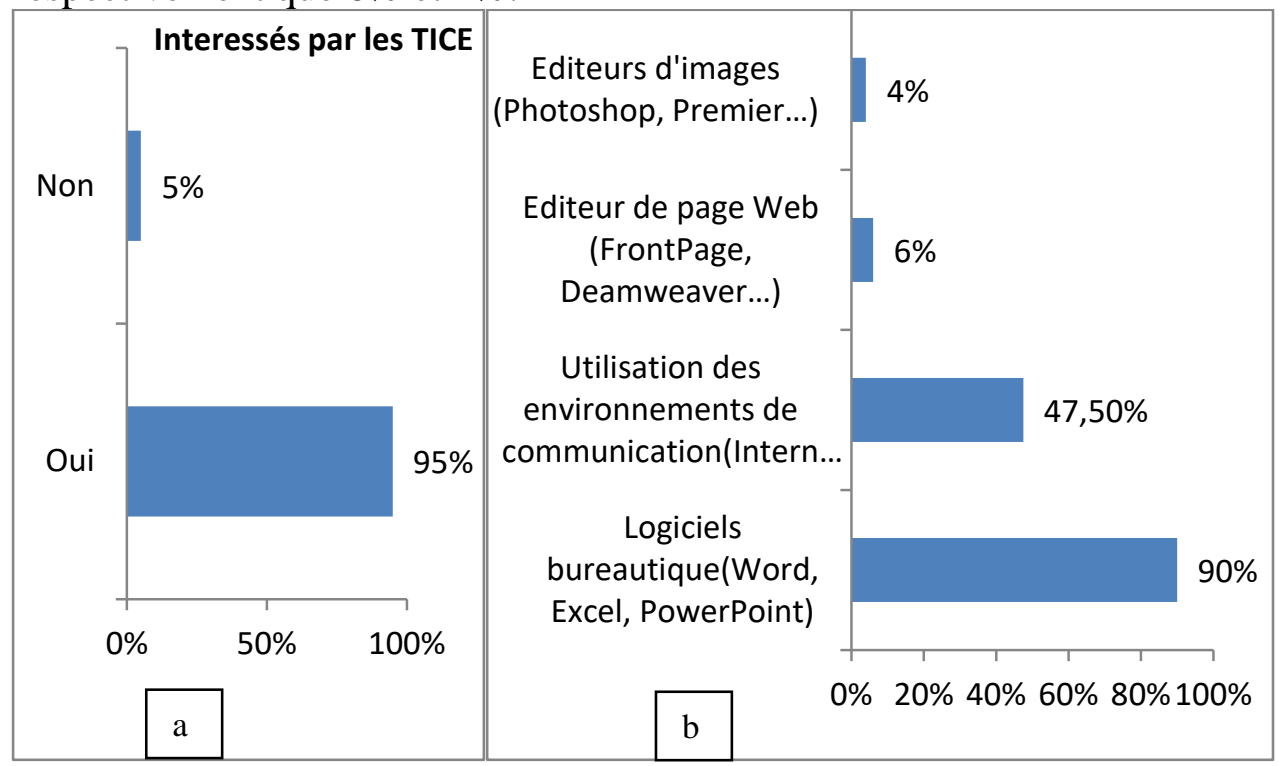

Figure 1 : usage personnel des enseignants interrogés concernant les TICE

\section{Usages professionnels des ressources numériques :}

Les résultats montrent que 20\% des enseignants questionnés n’ont jamais intégré les TICE dans l'enseignement, 57.5\% ont déclaré qu’ils les ont exploitées occasionnellement et $22.5 \%$ les ont utilisées d'une façon régulière (figure2a).

D’autre part, 57,5 \% des enseignants interrogés ont confirmé qu'ils ont une salle multimédia (salle GINIE) dans leurs établissements et ils sont équipés en ordinateurs (90\%) et vidéoprojecteurs (84,6\%). Malgré la 
présence plus ou moins importante des salles GINIE, on observe un faible pourcentage des enseignants (35\%) qui l'exploitent (figure2b).

Planifiez-vous des activités intégrantes les TICE dans votre

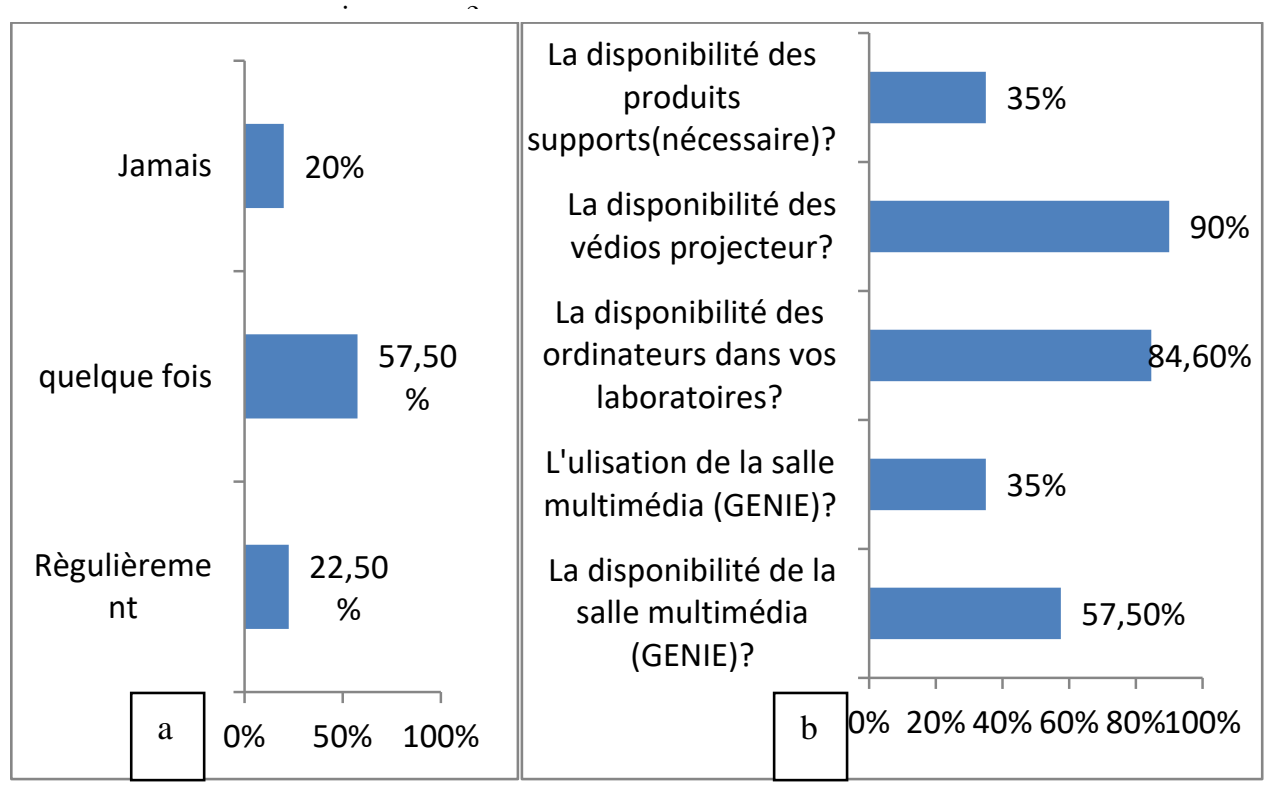

Figure 2 : usage des TICE, Infrastructures et environnement informatique dans les établissements

\section{Représentation des enseignants des STM sur l'utilité des TICE}

Les résultats montrent que $60 \%$ des enseignants jugent que l'intégration des TICE dans l'enseignement facilite l'opération d'apprentissage et $67.5 \%$ améliore la motivation des élèves et $52.5 \%$ considère que les TICE renforcent l'apprentissage, et 50 \% disent que les TICE aident à améliorer leur pédagogie.

$\mathrm{Au}$ cours de nos entretiens, les enseignants ont déclaré que l'utilisation des TICE aide à gagner du temps surtout dans la réalisation des dessins techniques, le fonctionnement des systèmes techniques et l'écriture du cours (constituant principalement des composants technologiques normalisés). S'ajoute à cela une forte motivation des élèves et beaucoup d'interaction surtout lors de la discussion de simulation, ce qui favorise aussi la construction de nouveaux concepts technologiques. 


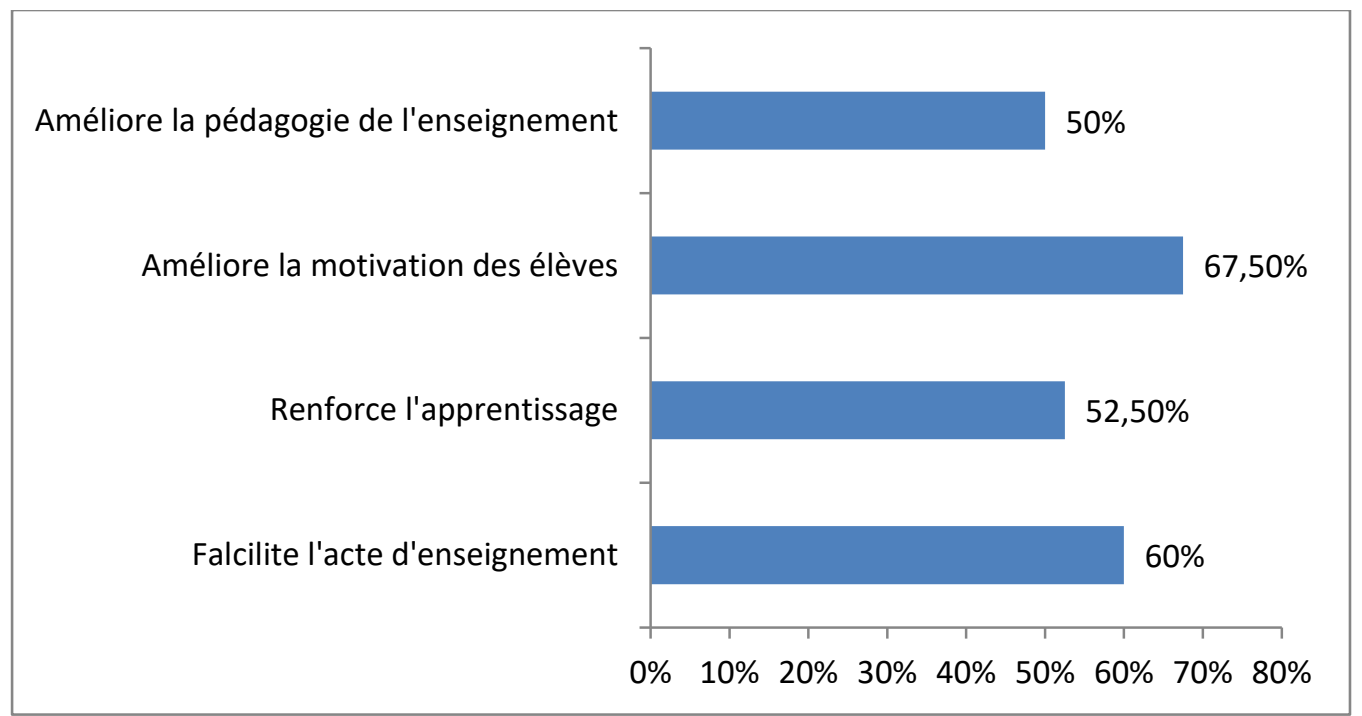

Figure 3 : Représentation des enseignants sur l'utilité des TICE

\section{Obstacles aux usages de TICE dans l'enseignement dans la filière STM}

La plupart des enseignants ont confirmé que le manque du temps (60\%), l'insuffisance d'équipements dans certains établissements (35\%) et le carence de formation dans l'intégration des TICE dans l'enseignement des SI de la filière STM (55\%) restent les grands obstacles aux usages des TICE dans la filière STM. D'autres problèmes s'ajoutent à cela tels que le surnombre des élèves, mais il reste présent avec un faible pourcentage (5\%), le manque de logiciels spécialisés et adaptés à la discipline représente un obstacle majore dans l'enseignement des SI (80\%) (figure3).

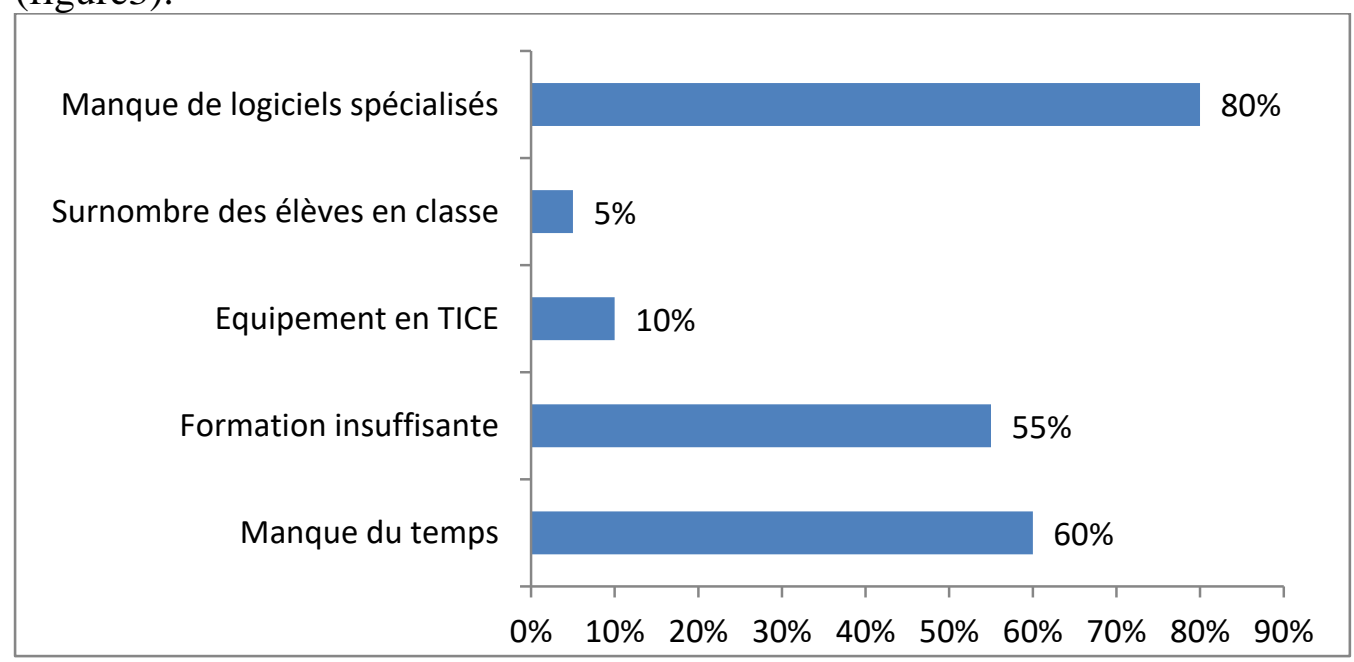

Figure 3 : obstacles aux usages des TICE dans la filière STM 


\section{Discussion}

Au terme de cette étude, on peut dire que les résultats obtenus nous permettent de répondre aux questions que nous avons formulées au départ. En effet, le travail a confirmé que la majorité des enseignants de sciences et technologies mécaniques présentent un intérêt remarquable concernant les TICE. Les attitudes de ces enseignants, leurs usages personnels relatifs aux TICE et même leur utilisation de ces technologies dans la préparation de leurs leçons sont encourageants.

Toutefois, l'intégration des TICE dans l'enseignement des sciences et technologies mécaniques en classe reste insuffisante. Ce fossé entre l'utilisation et l'intégration des TICE dans l'activité pédagogique de l'enseignant est dû à plusieurs contraintes, les plus importantes que nous avons détectées sont à classer en trois catégories.

La première catégorie porte sur l'insuffisance des équipements informatiques pour une réelle utilisation des TICE en tant que support pédagogique.

La deuxième a trait au manque en quantité, en qualité et en pertinence de ressources numériques éducatives (logiciels spécialisés, sites web) adaptées aux programmes enseignés.

La troisième concerne le manque de compétences technopédagogiques chez les enseignants interrogés, les résultats de notre étude montrent aussi que les problèmes de formation des enseignants liée à l'exécution du programme GENIE comptent parmi les principaux obstacles qui peuvent expliquer le fossé entre l'utilisation et l'intégration des TICE dans l'activité d’enseignement- apprentissage.

Parmi les problèmes liés à la réalisation du programme GENIE que nous avons relevés dans ce travail, on peut citer en particulier les suivants :

Les difficultés notoires au niveau des choix pertinents des scénarios pédagogiques (animation, vidéo, logiciel, expérience virtuelle, images...) et de leur planification alors que les formations encadrées par des enseignants de l'informatique ne répondent pas à la question suivante : Comment et quand intégrer les TICE dans l'enseignement des SI de la filière STM?

Il est à noter aussi que, dans le cadre du programme GENIE, la plupart des enseignants de la filière STM témoignent qu'ils n’ont pas bénéficié d'une formation sur l'intégration des TICE en relation avec la matière de la section STM. On préconise en conséquence de commencer par une analyse et définition des besoins en formation à travers une étude qui doit se faire antérieurement afin de rendre la formation exploitable par l'enseignant.

Pour réussir l'intégration des TICE dans le système éducatif marocain et en particulier dans l'enseignement des sciences et technologies mécaniques, nous formulons quelques recommandations qui nous paraissent 
urgentes telles que la nécessité de redynamiser le programme GENIE en l'élargissant en plusieurs salles dans le même établissement tout en s’intéressant à deux axes essentiels « motivation et formation des enseignants » et « développement des contenus ». L’implication des enseignants de la section dans le choix des formations appropriées et l'adoption de formations spécifiques pour l'intégration des TICE doivent être essentielles, surtout pour la STM. Cela pourrait motiver et intéresser les enseignants. Par ailleurs, il est nécessaire de mettre à la disposition des enseignants des ressources numériques normalisées et indexées adaptées aux programmes enseignés.

\section{Conclusion}

L'utilisation de l'outil informatique dans l'enseignement des SI dans la filière STM s'inscrit dans les champs des pratiques innovantes. Les résultats ont montré que la plupart des enseignants n’ont pas bénéficié d'une formation continue aux usages des TICE, adaptée à leurs besoins relatifs à la discipline, ce qui nuit aux choix pédagogiques de l'intégration des TICE dans l'enseignement des SI, car ils trouvent des difficultés au niveau des choix des scénarios pédagogiques (animation, vidéo, logiciel, expérience virtuelle, images...) et de leur planification. D’où la stratégie qui vise la généralisation des TICE dans l'enseignement marocain en général ne doit pas être articulée seulement autour des axes « infrastructure » et « développement de contenus », mais également autour de l’axe " formation des enseignants ».

On peut donc dire que les TICE représentent une solution pour rendre l'élève plus attentif, sérieux, qui reçoit des informations scientifiques et technologiques afin de s'engager entièrement dans le processus d'apprentissage. Le renforcement des équipements informatiques en dehors de la salle multimédia (GINIE), dans les laboratoires en particulier avec les logiciels spécialisés doit être pris en considération d’une manière continue afin de traduire les objectifs ciblés par le programme de SI de la filière STM.

\section{Abréviation : \\ TICE \\ STM \\ S.I \\ E.S \\ NTIC}

GENIE : GÉNéralisation des Technologies d'Information et de Communication dans l'Enseignement au Maroc.

CAO

FAO

: Conception Assistée par Ordinateur.

: Fabrication Assistée par Ordinateur.

CFAO : Conception et Fabrication Assistée par Ordinateur. 
GPAO : Gestion de Production Assistée par Ordinateur.

AREF : Académie Régionale de l'Education et de la Formation.

\section{References :}

1. A.Maouni, A. Minet, M. Khaddor, M. Madrane, M. Moune "L'intégration des TIC dans l'enseignement des SVT au Maroc: réalité et attentes » RADISMA, numéro 10(2014), 27 janvier 2014, disponible sur le site, www.radisma.info/document.php?id=1424.

2. F. Carugati, C. Tomasetto "Le corps enseignant face aux technologies de l'information et de la communication: un défi incontournable » Revue des sciences de l'éducation, vol $28, \mathrm{n}^{\circ} 2$, 2002 p305-324.

3. A.Biaz, A. Bennamara, A. Khyati, M. Talbi « Intégration des technologies de l'information et de la communication dans le travail enseignant, état des lieux et perspectives », 2009.

4. C.CLEARY1, A. AKKARI, D. CORTI «L'intégration des TIC dans l'enseignement secondaire », 2008.

5. R. Bibeau «Les Technologies de l'Information et de la Communication peuvent contribuer à améliorer les résultats scolaires des élèves ». EpiNet : Revue électronique de l'EPI, Paris, avril 2007.

6. S. Labrique, D. Grenier, F. Labrique « Entre savoir et savoir-être du rôle des TIC dans le processus d'apprentissage du génie électrique :compte rendu d’innovation », Didaskalia N²4-.2004.

7. T.Karsent, C.Gauthier « Les TIC bouleversent-elles réellement le travail des enseignants ? Formation et Profession, juin 2006.

8. O. ElOuidadi, A. Lakdim, K. Essafi, K. Sendide « Contribution à l'évaluation de l'impact de l'intégration des Tice dans l'enseignement et l'apprentissage : exemple de la division cellulaire (mitose) en deuxième année de baccalauréat sciences expérimentales ", revue électronique de l'EPI, Paris 2011, disponible sur le site: www.epi.asso.fr/revue/articles/a1110c.htlm.

9. Ministère de l'Education National et de la Formation Professionnelle Marocain, Charte nationale d'éducation et de formation, 1999.

10. Y. Elmadhi, B. Chiahou, D. Belghyti, K. Elkharim, H.Elhalouani «LES contraintes liées a l'intégration du TIC dans l'enseignement des sciences de la vie et de la terre au Maroc » European Scientific Journal 2014, Édition vol.10, No.34 ISSN: 1857 - 7881 (Print). Décembre 2014-Page 144

11. Ministère de l'Education National et de la Formation Professionnelle Marocain, Curriculum de sciences de l'ingénieur, sciences et technologies mécaniques, cycle de baccalauréat (1 ${ }^{\text {ère }}$ année et $2^{\text {ème }}$ année), Maroc, octobre 2006. 
12. K. Ahaji, A. Elhajjami, I. Laaziz « La politique d'intégration des Technologie de l'Information et de la Communication dans le Système éducatif marocain » 2013.

13. Ministère de l'Education National et de la Formation Professionnelle Marocain, Direction de la stratégie, des statistiques et de la planification, année scolaire 2016/2017.

Questionnaire sur l'intégration des TICE dans l'enseignement des SI de la filière STM.

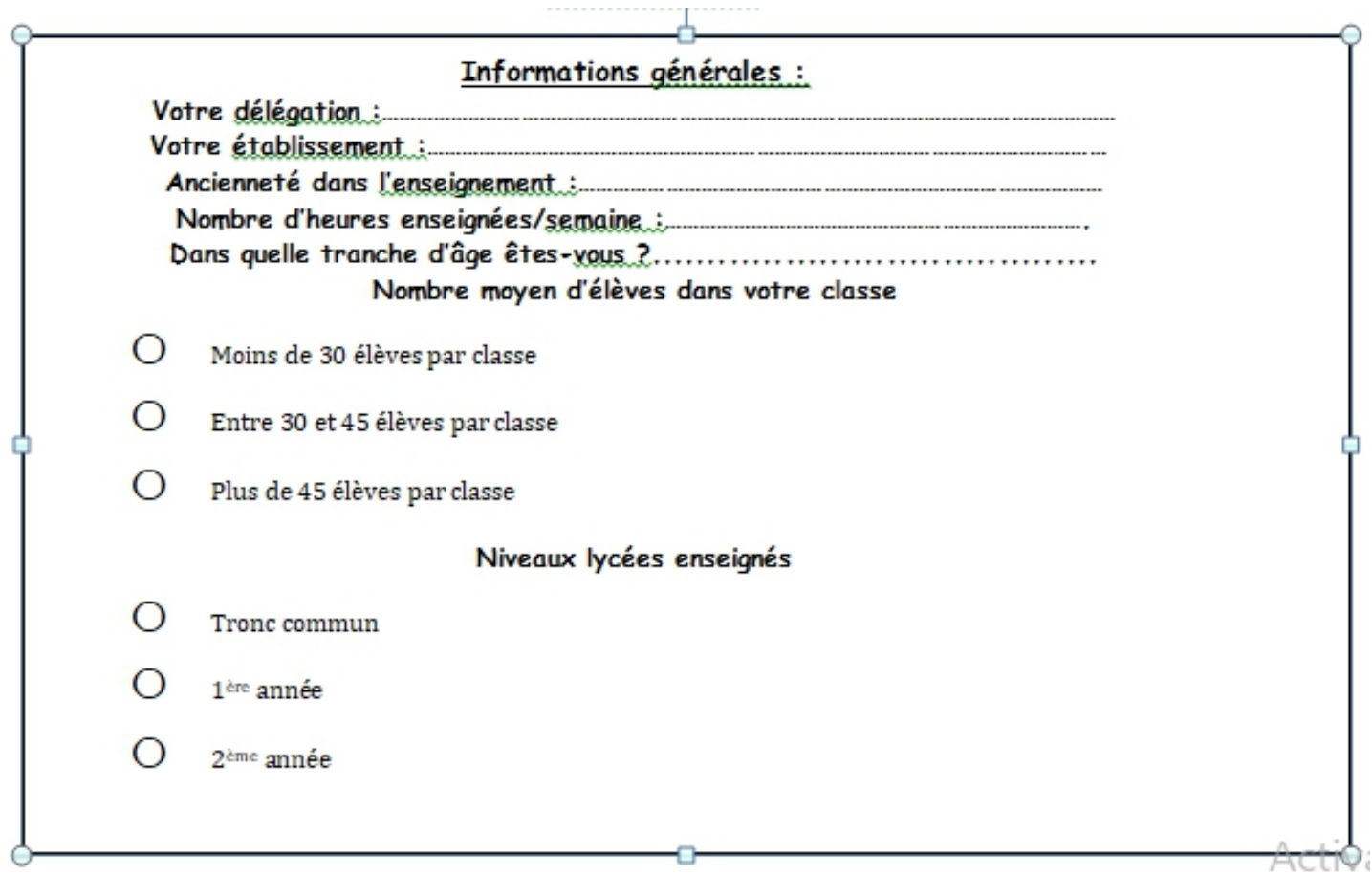




\section{A-Usages personnels des TICE:}

Maîtrisez-vous les applications suivantes?

Oui

$\mathrm{O}$ Non

Si oui indiquez les équipements informatiques que vous posséder.

\begin{tabular}{|c|c|c|}
\hline Applications & O & $\mathrm{N}$ \\
& $\mathrm{U}$ & $\mathbf{O}$ \\
& $\mathrm{I}$ & $\mathrm{N}$ \\
\hline Logiciels de bureautique (Word, Excel, PowerPoint) & & \\
\hline $\begin{array}{c}\text { Utilisation des environnements de communication (Internet, } \\
\text { courrier electronique, Forums...) }\end{array}$ & & \\
\hline Editeur de page Web (FrontPage, Dreamweaver, ...) & & \\
\hline Editeur d'images (Photoshop, Premier...) & & \\
\hline
\end{tabular}

B-Usages professionnels des ressources numériques et environnement

\section{informatique dans l'établissement:}

Organisez vous des activités intégrantes les TICE dans votre enseignement?

Régulièrement

Quelquefois

Jamais

Avez-vous une salle multimédia dans votre établissement (salle GINIE)?

Oui

Non

Utilisez-vous la salle multimédia (GENIE)?

Oui

Non

Avez-vous un vidéoprojecteur?

O Oui

Non

Avez-vous des produits supports (nécessaire)?

Oui

Non 
European Scientific Journal May 2017 edition Vol.13, No.13 ISSN: 1857 - 7881 (Print) e - ISSN 1857- 7431

Avez-vous suffisamment d'ordinateurs dans vos laboratoires?

Oui

Non

C-Représentation des enseignants sur l'utilité des TICE:

Que pensez-vous de l'utilité des TICE dans vos pratiques pédagogiques ?

Facilite la tâche

Renforce l'apprentissage

Améliore la motivation des élèves

Améliore la pédagogie de l'enseignement

D-Obstacles à l'usage des TICE dans la filière STM:

Quelles sont les causes qui entravent l'usage des TICE pour votre enseignement ?

Manque de temps

Formation insuffisante

Equipements informatiques

Manque de logiciels spécialisés

Surnombre des élèves en classe

491 